\title{
Self-assembled Au nanoparticles on heated Corning glass by dc magnetron sputtering: size-dependent surface plasmon resonance tuning
}

\author{
S. Grammatikopoulos, ${ }^{1}$ S. D. Pappas, ${ }^{1}$ V. Dracopoulos, ${ }^{2}$ P. Poulopoulos,,${ }^{1,3,4, *}$ \\ P. Fumagalli, ${ }^{4}$ M.J. Velgakis ${ }^{1}$ and C. Politis, ${ }^{1,5}$ \\ ${ }^{1}$ Laboratory of High-Tech Materials, School of Engineering, University of Patras, 26504 Patras, \\ Greece \\ ${ }^{2}$ Foundation for Research and Technology, Hellas-Institute of Chemical Engineering and High \\ Temperature Chemical Processes, (FORTH/ICE-HT), P.O. Box 1414, 26504 Patras, Greece \\ ${ }^{3}$ Materials Science Department, University of Patras, 26504 Patras, Greece \\ ${ }^{4}$ Institut für Experimentalphysik, Freie Universität Berlin, Arnimallee 14, D-14195 Berlin-Dahlem, \\ Germany \\ ${ }^{5}$ Department of Materials Science and Engineering, University of Texas at Arlington, Arlington, TX \\ 76019, USA
}

\begin{abstract}
:
We report on the growth of Au nanoparticles on Corning glass by direct current magnetron sputtering and on the optical absorption of the films. The substrate temperature was kept to relatively high temperatures of $100{ }^{\circ} \mathrm{C}$ or $450{ }^{\circ} \mathrm{C}$. This lead to the growth of Au nanoparticles instead of smooth Au films as the surface energy of Au is much larger than the one of glass. The size of the particles depended on the substrate temperature and deposition time and was shown to follow a logarithmic normal distribution function. Both, the surface plasmon resonance position and bandwidth, were found to depend upon the average particle size. The surface plasmon resonance position showed a $75 \mathrm{~nm}$ continuous blue shift from $14 \mathrm{~nm}$ down to $2.5 \mathrm{~nm}$ average particle size. Thus, we have shown how to tune the nanoparticle size and surface plasmon resonance of $\mathrm{Au}$ by varying the substrate temperature and deposition time. The experimental results are reproduced reasonably using a method which is based on the size- and wavelength-dependent complex dielectric function of Au within the framework of the Mie theory for the optical properties of metallic nanospheres.
\end{abstract}

Keywords: Au, nanoparticles, magnetron sputtering, optical properties, absorption, surface plasmon resonance

* Corresponding author: P. Poulopoulos, Tel. +30-2610-997884, Fax +30-2610-997255 email: poulop@upatras.gr 


\section{Introduction}

Nanostructuring of metals results in the appearance of surface plasmon resonances (SPR), which are strong collective oscillations of the free electrons on the surface of the conducting nanostructures (Mie 1908). These give rise to peaks in the light absorption spectra of the materials, which depend on the nanostructure geometry and on the dielectric properties of the metal and the surrounding material. Many applications are related to SPR, like fabrication of energy efficient coatings, involvement in cancer therapy, biological imaging and biomedicine, cholesterol sensing, lithography, surface enhanced Raman spectroscopy, fabrication of negative permittivity near-field superlenses etc. (Link and ElSayed 1999; Jain et al. 2006; Saxena and Goswami 2012; Grochowska et al. 2011). The energy position of the SPR and its bandwidth depend on the size of the nanoparticles. Decreasing of the size broadens the SPR, while it may result in blue or blue and red shift of the SPR position (Kreibig and Genzel 1985). In the case of Au, nanostructuring is usually achieved through the formation of colloidal solutions the color of which depends on the particle size (Link and El-Sayed 1999; Wand and Gunasekaran 2012), or in solid state by high temperature annealing $\left(400-800{ }^{\circ} \mathrm{C}\right)$ (Tesler at al. 2011; Romani et al. 2012), irradiation by short ultraviolet (UV) laser pulses of smooth Au films (Grochowska et al. 2011) and by using gas aggregation sources or ion cluster sourced (Heer 1993).

In this work, we prepare Au nanoparticles via an alternative method: namely, we maintain the substrate temperature higher than the room temperature (RT). Gold does not wet easily Corning glass or the native silicon oxide, due to the large difference in the surface

energies, $1.5 \mathrm{~J} / \mathrm{m}^{2}$ for $\mathrm{Au}$ (Vitos et al. 1998), and only $0.3 \mathrm{~J} / \mathrm{m}^{2}$ for $\mathrm{SiO}_{2}$ (Wu et al. 2007). Moreover, by increasing the temperature the system is driven closer to thermodynamic equilibrium, that is, isolated Au islands/particles are easily formed on glass. The surface of the Corning glass with the $\mathrm{Au}$ nanoparticles is monitored via high resolution Scanning 
Electron Microscopy (SEM). The particle size follows a logarithmic normal (log-norm) distribution function. The average size ranges between 2.5 and $14 \mathrm{~nm}$ for six different preparation settings. The position of the SPR is found to present blue shift as the average nanoparticle size decreases. On the other hand, the SPR bandwidth initially decreases and then saturates to about $100-110 \mathrm{~nm}$. Our work shows an alternative growth method for $\mathrm{Au}$ nanoparticle formation by varying the substrate temperature resulting in controllable nanoparticle size and tuning of the SPR of the films. While, the vast majority of papers which deal with Au nanoparticles on various substrates include two preparation steps: (i) Au films are deposited by Physical Vapor Deposition methods on substrates and (ii) post annealing of the films under various inert atmospheres is performed in order the continuous films to break into islands. The island shape may be irregular unless the annealing is performed at very high temperatures. In our case, the deposition temperature is kept in relatively high values. This allows the particles to take easier their final equilibrium nearly spherical shape (Vasisht and Shirokoff 2010). The deposition temperature is more effective than the same temperature of annealing continuous films; even relatively high deposition temperature allows the sample to obtain the equilibrium shape immediately during deposition without the need to overcome thermodynamic diffusion barriers that may be introduced when a continuous or semi-continuous film is formed at low deposition temperatures. We think that the uniformity in shape of Au nanoparticles prepared at $450{ }^{\circ} \mathrm{C}$ results in SPR with almost constant narrow bandwidth.

\section{Experimental Section}

Au nanoparticles were deposited on Corning glass with the help of a Balzers SCD 040 direct current (dc) magnetron sputtering apparatus (sputter coater). The base pressure of the chamber was $4 \times 10^{-3}$ mbar. The argon pressure during the sputtering procedure was kept 
constant at $5 \times 10^{-2}$ mbar with the help of a fine valve. The sputter coater power was equal to $\sim 3 \mathrm{~W}(195 \mathrm{~V} \times 0.015 \mathrm{~A})$. During the deposition the substrate temperature was kept constant via a temperature controller. Several samples were prepared in the range of $100-450{ }^{\circ} \mathrm{C}$.

X-ray reflectivity (XRR) measurements were recorded via a standard powder diffractometer (SIEMENS-BERTHOLD) with a Ni-filtered $\mathrm{CuKa}_{1}$ radiation $(\lambda=0.15418$ $\mathrm{nm})$. The deposition rates were calibrated with the help of Kiessig fringes appearing in the XRR patterns of smooth Au films grown at RT (Kiessig 1931). Such fringes appear for example in the XRR pattern of a $21.5 \mathrm{~nm}$ Au film on Corning glass, see Fig. 1. From the period of the fringes one can determine the film thickness within an accuracy of $\pm 5 \%$ (Poulopoulos et al. 2003). The deposition rate was found to be $0.48 \mathrm{~nm} / \mathrm{s}$. Our discontinuous $\mathrm{Au}$ films were grown successively at elevated substrate temperatures with a nominal thickness $\mathrm{t}$ in the range of $0.5-3 \mathrm{~nm}$. By the term 'nominal thickness' we mean the thickness of a smooth Au film which would grow in the same deposition time at RT. Such smooth films are shown, for example by Karoutsos et al. (2012). However, in the present work, due to higher substrate temperature, our films are discontinuous in the form of $\mathrm{Au}$ nanoparticles on Corning glass.

The surface morphology of selected samples was studied via SEM. The SEM analysis was carried out in a Zeiss SUPRA 35VP electron microscope, with a point resolution of $1.7 \mathrm{~nm}$ at $20 \mathrm{kV}$. Finally, the UV - visible spectra were recorded at RT in transmission geometry with a Perkin-Elmer Lambda-35 UV-visible photospectrometer apparatus in the wavelength range of $200-1100 \mathrm{~nm}$.

\section{Results and Discussion}

In Fig. 2, six SEM images of gold nanoparticles prepared under different conditions on Corning glass are shown. The films have different nominal thickness and substrate 
temperature during deposition. The size and morphology of the gold nanoparticles is found to depend on these two parameters. In Figs. 2 (a,b,c), the substrate temperature during the deposition was $100^{\circ} \mathrm{C}$, while the nominal thickness was $2.4,1.5$, and $0.5 \mathrm{~nm}$, respectively. In Figs. 2(b,c), we observe the formation of very small non-agglomerated nanoparticles with small distances between them. However, in the case of the $2.4 \mathrm{~nm}$ thick film, Fig. 2(a), sometimes gold nanoparticles merge giving rise to larger islands. In Fig. 2(d,e,f), on the other hand, we have the same nominal Au-film thickness with Figs. 2(a,b,c), respectively, but the substrate temperature was kept at $450^{\circ} \mathrm{C}$. Comparison of the corresponding images reveals that for the same nominal film thickness, the nanoparticles are smaller when grown under the higher substrate temperature. In Fig. 2(f), we have the smallest nanoparticles of gold.

In order to quantify the information of Fig. 2, we have created for each SEM image one nanoparticle-size distribution column-bar diagram. To get adequate statistical results, we have taken a random sample of at least 200 islands for each SEM image. The solid line in each column-bar diagram is the best fitted log-normal function (Escobar et al. 1985; Karoutsos et al. 2007):

$$
f(x)=\frac{k}{\sigma \sqrt{2 \pi}} \cdot e^{\left[-\frac{(\ln x-\ln d)^{2}}{2 \sigma^{2}}\right]}
$$

In Eq. (1), $\mathrm{d}$ is the average nanoparticle size (diameter), $\mathrm{k}$ is a normalization constant and $\sigma$ is the standard deviation of $\ln (\mathrm{x})$. As we can see in Fig. 3, the average nanoparticle size varies between 2.5 and $14 \mathrm{~nm}$. In order to be able to compare the relative variation of the $\log$ normal distribution functions, we have divided the $\mathrm{x}$-axis in each of the diagrams by the average nanoparticle diameter $\mathrm{d}$ of it, so that the maximum always appears at $\mathrm{x}=1$ and kept 
the same $x$-scale length from 0 to 3.5 . More or less all distributions look similarly broad except the one of Fig. 3(a), which appears slightly broader. This might be due to the fact that it contains some larger agglomerated particles. Table 1 contains the nominal film thickness, the average nanoparticle size and the the standard deviation of the fits for the six samples of Figs. 2 and 3. The standard deviations, surprisingly including the one of the distribution of Fig. 3(a), are within $10 \%$ equal to about 2 , reflecting the similarity in the appearance of the $\log$ normal distribution curves of Fig. 3.

In Fig. 4, the normalized light absorption spectra of our six discontinuous Au films are shown. By normalization, (i) we divide the transparency $\mathrm{T}$ of the films with the $\mathrm{T}_{\mathrm{S}}$ of the substrate, (ii) we use the formula: absorbance $=-\log \left(\mathrm{T} / \mathrm{T}_{\mathrm{S}}\right)$ and (iii) we scale the absorbance value at energies far below the SPR to 0 and at the SPR peak to 1 (Garcia et al. 2005; Wang et al. 2008). Then, we vertically shift the spectra for clarity of presentation. Corning glass becomes opaque after $4 \mathrm{eV}$, however, it is fully transparent at the whole energy range of the measurement between $1.1-3 \mathrm{eV}$. The signal-to-noise ratio is very high even for the thinnest sample. In the inset of Fig. 4 we present the absorbance of the continuous Au film. The spectrum, which is characteristic for continuous Au films (Romani et al. 2012), is distinctly different from the ones of the discontinuous samples.

All our discontinuous films show a clear SPR at energies $2-2.5 \mathrm{eV}$. Its position is blue shifted from $604.5 \mathrm{~nm}$ to $530 \mathrm{~nm}$ as the average nanoparticle size decreases from 14 to $2.5 \mathrm{~nm}$. The bandwidth or full width at half maximum (FWHM) of the SPR of our films initially decreases with decreasing average nanoparticle size and then remains almost constant at values of about $100-110 \mathrm{~nm}$. The results are presented in Fig. 5 and tabulated in Table 1. Concerning the position of the SPR, there are reports showing blue shift with decreasing nominal thickness and/or particle size, e.g. by Tesler et al. (2001), or no shift at all, e.g. by Garcia et al. (2005). Safe conclusions can be drawn only if the Au nanoparticles 
are monodisperse with a very narrow size distribution. Scaffardi et al. (2005) have demonstrated by experiment and theory that for Au nanoparticles with radius beyond $10 \mathrm{~nm}$, the absorption peak broadens and shifts to higher wavelengths. This justifies our results for our samples a and b where islands with large sizes are present in the log normal distribution, Figs 3(a,b). Then, Scaffardi et al. (2005) showed that absorption spectra by particles with radii between about 2.6 and $10 \mathrm{~nm}$ have a SPR peak near $520 \mathrm{~nm}$ and they are independent of size. Below $2.6 \mathrm{~nm}$, the spectra again show dependence with radius: peaks remain near $520 \mathrm{~nm}$ but the contrast between the maximum and the minimum near $440 \mathrm{~nm}$ depends clearly on size. While these notes should hold for monodisperse nanoparticles, the mixture of many sizes in our samples, see Figs. 3(c-d), should be responsible for the differences observed between our data and the ones of Scaffardi et al. (2005).

Scaffardi et al. (2005) provides also the size and wavelength dependence of the real $n$ and imaginary $\mathrm{k}$ parts of the complex refractive index $\mathrm{N}=\mathrm{n}+\mathrm{ik}$ of gold. With the help of them, one may calculate the size and wavelength dependence of the complex dielectric function $\varepsilon=\varepsilon_{1}+\mathrm{i} \varepsilon_{2}$ via the relations: $\varepsilon_{1}=\mathrm{n}^{2}-\mathrm{k}^{2}$ and $\varepsilon_{2}=2 \mathrm{nk}$. In this case, the size- and wavelength-dependent absorbance $\mathrm{Q}_{\mathrm{abs}}$ of $\mathrm{Au}$ nanoparticles, the shape of which is approximated by nanospheres (this is reasonable at least for samples b-f as Fig. 2 reveals), can be calculated via the Mie theory (Mie 1908; Blaber et al. 2007; Hsieh et al. 2011):

$$
\mathrm{Q}_{\mathrm{abs}}=(24 \pi \mathrm{r} / \lambda) \cdot\left(\varepsilon_{\mathrm{m}}^{3 / 2} \varepsilon_{2} /\left[\varepsilon_{2}^{2}+\left(\varepsilon_{1}+2 \varepsilon_{\mathrm{m}}\right)^{2}\right]\right.
$$

where $\varepsilon_{\mathrm{m}}$ stands for the dielectric constant of the surrounding medium. In our case it should be between 1 (air) and $3.9\left(\mathrm{SiO}_{2}\right.$ - as Corning glass is about $\left.94 \% \mathrm{SiO}_{2}\right)$. This theoretical approach is valid for spherical particles with a relatively small filling factor $\mathrm{f}$ (when placed in a dielectric medium) or small areal density a (when placed on a substrate as in our case). 
Indeed, Takele et al. (2006) have demonstrated that up to f values of about $25 \%$ the SPR position remains constant. Further increments in the filling factor increase the cluster/nanoparticle size beyond $10 \mathrm{~nm}$ and the interparticle distance is comparable with the cluster size. Thus, further increase of $\mathrm{f}$ up to the percolation threshold leads to a red shift of the plasmon resonance due to electrodynamic interactions between the particles at a low particle separation (Takele et al. 2006). When $\mathrm{f}$ is considerable, calculations for the SPR should better follow the Maxwell-Garnett theory, as shown, for example, by Salz et al. (1999) or Xu et al. (2005). Table 1 reveals that the areal density a of our samples varies between $13.7 \%-52.7 \%$ and therefore Eq. (2) should hold better only for the four samples with a $<25 \%$. Moreover, the series of samples grown at the lower temperature of $100{ }^{\circ} \mathrm{C}$ may have particle shapes that deviate from the thermodynamic equilibrium shape, which should be spherical (Vasisht and Shirokoff 2010). Therefore we attempted calculations only for the high deposition temperature samples.

Since in our case we do not have monodisperse nanoparticles, we decided to make an alteration of the method of Scaffardi et al. (2005) and fit the experimental data with the help of Eq. (2) and Fig. 3. That is, we considered the $\mathrm{Q}_{\mathrm{abs}}$ for a sample to be the weighted average of the $\mathrm{Q}_{\mathrm{abs}}$ of the various particle sizes appearing in the log-norm distribution of each film. The total calculated $\mathrm{Q}_{\mathrm{abs}}$ is then scaled to unity in order to be comparable to the experiment. The result for the sample e is presented in Fig. 6 (dashed line). For this calculation, $\varepsilon_{\mathrm{m}}$ was taken equal to 3 , which is a reasonable value as aforementioned. One may see that the SPR peak position of the experimental data (open symbols) is nicely reproduced, however, the calculated peak is by about $20 \%$ narrower than the experimental one. Broadening of the calculated spectrum can be obtained by using a larger amount of smaller particles than the ones of Fig. 3(e). If for example the average diameter is not $3.84 \mathrm{~nm}$ but about $2.7 \mathrm{~nm}$, the calculation provides the solid line which fits better to the experimental data regarding the 
bandwidth. The difference between experiment and calculation could be due to the fact that our island size is very close to the point resolution of our SEM device or due to limitations of the model for calculation.

\section{Conclusions}

In this work, we have shown how to tune the position and the bandwidth of the surface plasmon resonances of $\mathrm{Au}$ by depositing the material at elevated temperatures; this results in the direct formation of tiny Au nanoparticles. The island size follows a logarithmic normal distribution function. It was found that deposition of $\mathrm{Au}$ at $450^{\circ} \mathrm{C}$ results in the production of smaller nanoparticle and the optical absorption spectra reveal narrower bandwidth as compared to samples produced at $100^{\circ} \mathrm{C}$ substrate temperature. On the other hand, the position of the surface plasmon resonance shows a monotonous decrease with decreasing nanoparticle size irrespective of deposition temperature. Our experimental results were reproduced fairly well with a calculation method based on the size- and wavelengthdependent complex dielectric function of Au within the framework of the Mie theory for the optical properties of metallic nanospheres (Mie 1908).

\section{Acknowledgements}

This work has been supported by the Research Committee of the University of Patras, grant Karatheodori, Nr. C.905/2009. One of us (P.P.) acknowledges the Center for International Cooperation (C.I.C.) of the Freie Universität Berlin for partial financial support and the Institute for Experimental Physics, Freie Universität Berlin for their kind hospitality. Last, but not least, we would like to thank the TSMEDE for systematically providing us financial support for our research. 


\section{References}

Blaber MG, Arnold MD, Harris N, Ford MJ, Cortie MB (2007) Plasmon absorption in nanospheres: A comparison of sodium, potassium, aluminium, silver and gold. Physica B 394:184-187

Escobar MA, Magana LF, Valenzuela R (1985) Effect of the grain size distribution on the magnetization curve. J Appl Phys 57:2142-2146

Garcia MA, de la Venta J, Crespo P, LLopis J, Penadés S, Fernández A, Hernando A (2005) Surface plasmon resonance of capped Au nanoparticles. Phys Rev B 72: 241403(R)

Grochowska K, Nedyalkov N, Atanasov P, Sliwinski G (2011) Nanostructuring of thin Au films by means of short UV laser pulses. Opto-Electronics Rev 19(3)327-332

Heer W.A. de (1993) The physics of simple metal clusters: experimental aspects and simple models. Rev. Mod. Phys. 65: 611-676 and references therein

Hsieh JH, Li Chuan, Wu YY, Jang SC (2011) Optical studies on sputter-deposited Ag-SiO nanoparticle composites. Thin Solid Films 519:7124-7128

Jain PK, Lee KS, El-Sayed IH, El-Sayed MA (2006) Calculated Absorption and Scattering Properties of Gold Nanoparticles of Different Size, Shape, and Composition: Applications in Biological Imaging and Biomedicine. J Phys Chem B 110:7238-7248

Karoutsos V, Papasotiriou P, Poulopoulos P, Kapaklis V, Politis C, Angelakeris M, Kehagias T, Flevaris NK, Papaioannou ET (2007) Growth modes of nanocrystalline Ni/Pt multilayers with deposition temperature. J Appl Phys 102:043525

Karoutsos V, Toudas M, Delimitis A, Grammatikopoulos S, Poulopoulos P (2012) Microstructural evolution in nanostructured gold films. Thin Solid Films 520:40744079

Kiessig H (1931) Untersuchungen zur Totalreflexion von Röntgenstrahlen. Ann Physik (Leipzig) 402:715 
Kreibig U, Genzel L (1985) Optical absorption of small metallic particles. Surf. Sci. $156: 678-700$

Link S, El-Sayed MA (1999) Size and Temperature Dependence of the Plasmon Absorption of Colloidal Gold Nanoparticles. J Phys Chem B 103:4212-4217 (1999)

Mie G, (1908) Beiträge zur Optik trüber Medien, speziell kolloidaler Metallösungen. Ann Physik 25(3):377-445

Poulopoulos P, Angelakeris M, Papaioannou ET, Flevaris NK, Niarchos D, Nyvlt M, Prosser V, Visnovsky S, Mueller C, Fumagalli P, Wilhelm F, Rogalev A (2003) Structural, magnetic, and spectroscopic magneto-optical properties aspects of $\mathrm{Pt}-\mathrm{Co}$ multilayers with intentionally alloyed layers. J Appl Phys 94(12): $7662-7669$

Romani EC, Vitoreti D, Gouvea PMP, Caldas PG, Prioli R, Paciornik S, Fokine M, Braga AMB, Gomes ASL, Carvalho ICS (2012) Gold nanoparticles on the surface of sodalime glass: morphological, linear and nonlinear optical characterization. Optics Express 20(5): 5429-5439

Salz D, Lamber R, Wark M, Baalmann A, Jaeger N (1999) Metal clusters in plasma polymer matrices. Phys Chem Chem Phys 1:4447-4451

Saxena U, Goswami P (2012) Electrical and optical properties of gold nanoparticles: applications in gold nanoparticles-cholesterol oxidase integrated systems for cholesterol sensing. J Nanopart Res 14:813

Scaffardi LB, Pelegri N, De Sanctis O, Tocho JO (2005) Sizing gold nanoparticles by optical extinction spectroscopy. Nanotechnology 16:158-163

Takele H, Greve H, Pochstein C, Zaporojtchenko V, Faupel F, (2006) Plasmonic properties of Ag nanoclusters in various polymer matrices. Nanotechnology 17:3499-3505 
Tesler AB, Chuntonov L, Karakouz T, Bendikov TA, Haran G, Vaskevich A, Rubinstein I (2011) Tunable Localized Plasmon Transducers Prepared by Thermal Dewetting of Percolated Evaporated Gold Films. J Phys Chem C 115:24642-24652

Vasisht S., Shirokoff J. (2010) Preferred orientations in nano-gold/silica/silicon interfaces. Appl. Surf. Sci. 256: 4915-4923

Vitos L, Ruban AV, Skriver HL, Kollár J (1998) The surface energy of Metals. Surf Sci 411: 186-202

Wang Chang-Hai, Liu Chi-Jen, Wang Cheng-Liang, Hua Tzu-En, Obliosca Judy M, Lee KH, Hwu Y, Yang Chung-Shi, Liu Ru-Shi, Lin Hong-Ming, Je Jung-Ho, Margaritondo G (2008) Optimizing the size and surface properties of polyethylene glycol (PEG)gold nanoparticles by intense x-ray irradiation J Phys D: Appl Phys 41:195301

Wang YC, Gunasekaran S (2012) Spectroscopic and microscopic investigation of gold nanoparticle nucleation and growth mechanisms using gelatin as a stabilizer. J Nanopart Res 14:1200

Wu Yun-Chung, Wang Liang-Wei, Lai Chih-Huang (2007) Low temperature ordering of (001) granular FePt films by inserting ultrathin $\mathrm{SiO}_{2}$ layers. Appl. Phys. Lett. 91: 072502

Xu G, Tazawa M, Jin P, Nakao S (2005) Surface plasmon resonance of sputtered Ag films: substrate and mass thickness dependence. Appl Phys A80:1535-1540 


\section{Figure Captions}

Fig. 1

XRR pattern of a smooth Au film grown at RT on Corning glass. The characteristic oscillations (Kiessig fringes) indicate the formation of a uniform continuous film with a well-defined thickness. From the relative positions of the Kiessig fringes the film thickness was determined. The preparation conditions are also provided on the Figure.

Fig. 2 (Please use 2 columns)

SEM images of six deposited Au thin films, $t$ is the nominal thickness of film and $\mathrm{T}$ the substrate temperature during deposition: Sample (a) $t=2.4 \mathrm{~nm}, \mathrm{~T}=100^{\circ} \mathrm{C}$, sample (b) $\mathrm{t}=1.5$ $\mathrm{nm}, \mathrm{T}=100^{\circ} \mathrm{C}$, sample $(\mathrm{c}) \mathrm{t}=0.5 \mathrm{~nm}, \mathrm{~T}=100^{\circ} \mathrm{C}$, sample $(\mathrm{d}) \mathrm{t}=2.4 \mathrm{~nm}, \mathrm{~T}=450^{\circ} \mathrm{C}$, sample (e) $\mathrm{t}=1.5 \mathrm{~nm}, \mathrm{~T}=450^{\circ} \mathrm{C}$, sample (f) $\mathrm{t}=0.5 \mathrm{~nm}, \mathrm{~T}=450^{\circ} \mathrm{C}$.

Fig. 3 (Color online - Please use 2 columns)

Nanoparticle (grain) - size distribution diagrams for discontinuous Au thin films. Each diagram corresponds to an image of Fig. 2. $\mathrm{d}$ is the average particle size obtained by lognorm fitting (solid lines).

Fig. 4 (Color online)

UV-VIS normalized absorption spectra for samples of various average nanoparticle size and substrate temperature. The inset graph shows the $21.5 \mathrm{~nm}$ thick Au film as a reference for a smooth film deposited at RT. 


\section{Fig. 5 (Color online)}

Dependence of the SPR position on the average nanoparticle size. A systematic blue shift is observed with decreasing nanoparticle size. The inset shows the corresponding dependence of the SPR bandwidth (FWHM). Filled (open) symbols corresponds to substrate temperature during deposition of $100^{\circ} \mathrm{C}\left(450^{\circ} \mathrm{C}\right)$.

Fig. 6 (Color online)

Experimental spectrum for the normalized absorbance (open symbols) and two calculated results taking into account the real log-norm distribution function for sample e (calculation 1) or a hypothetical one with $30 \%$ smaller particle size (calculation 2). 
TABLE 1 Average nanoparticle size $d$, standard deviation $\sigma$, areal density a, absorbance peak position and FWHM of all samples with different nominal thickness $t$ and substrate temperature. In samples a-c (d-f) the substrate temperature was $100^{\circ} \mathrm{C}\left(450^{\circ} \mathrm{C}\right)$.

Sample t $(\mathrm{nm}) \quad \mathrm{d}(\mathrm{nm}) \quad \sigma \quad \mathrm{a}(\%) \quad \mathrm{SPR}(\mathrm{eV})-(\mathrm{nm}) \quad$ FWHM $(\mathrm{nm})$

\begin{tabular}{|c|c|c|c|c|c|c|}
\hline $\mathrm{a}$ & 2.4 & 14.0 & 1.81 & 17.1 & $2.05-604.5$ & 152.0 \\
$\mathrm{~b}$ & 1.5 & 6.6 & 1.92 & 52.7 & $2.14-582.5$ & 129.0 \\
$\mathrm{c}$ & 0.5 & 3.80 & 2.01 & 13.7 & $2.27-547.1$ & 116.6 \\
\hline $\mathrm{d}$ & 2.4 & 5.27 & 2.23 & 34.4 & $2.21-560.5$ & 103.3 \\
$\mathrm{f}$ & 1.5 & 3.84 & 2.22 & 23.7 & $2.25-550.8$ & 101.6 \\
$\mathrm{e}$ & 0.5 & 2.54 & 2.22 & 13.4 & $2.34-530.0$ & 107.9 \\
\hline
\end{tabular}




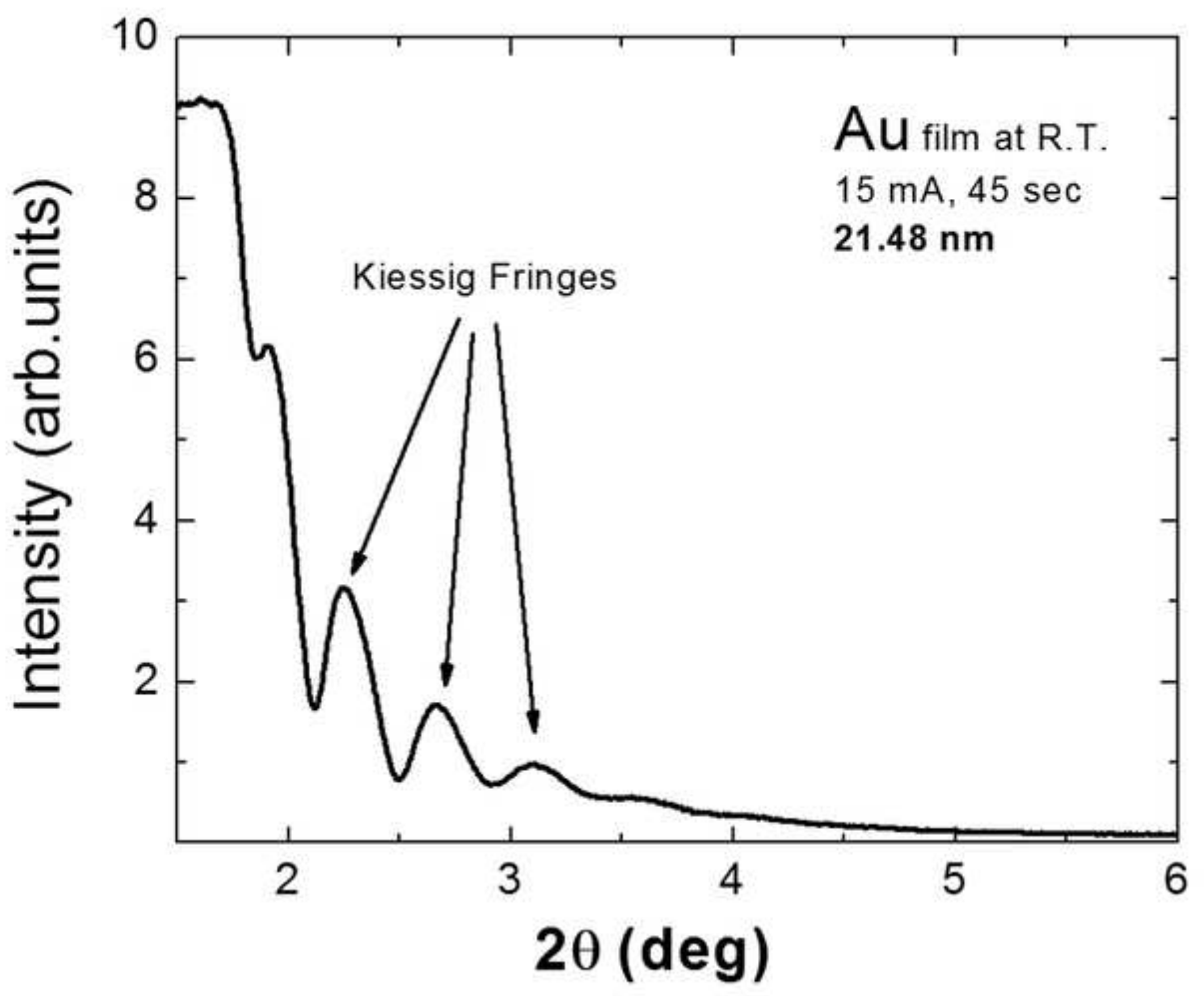



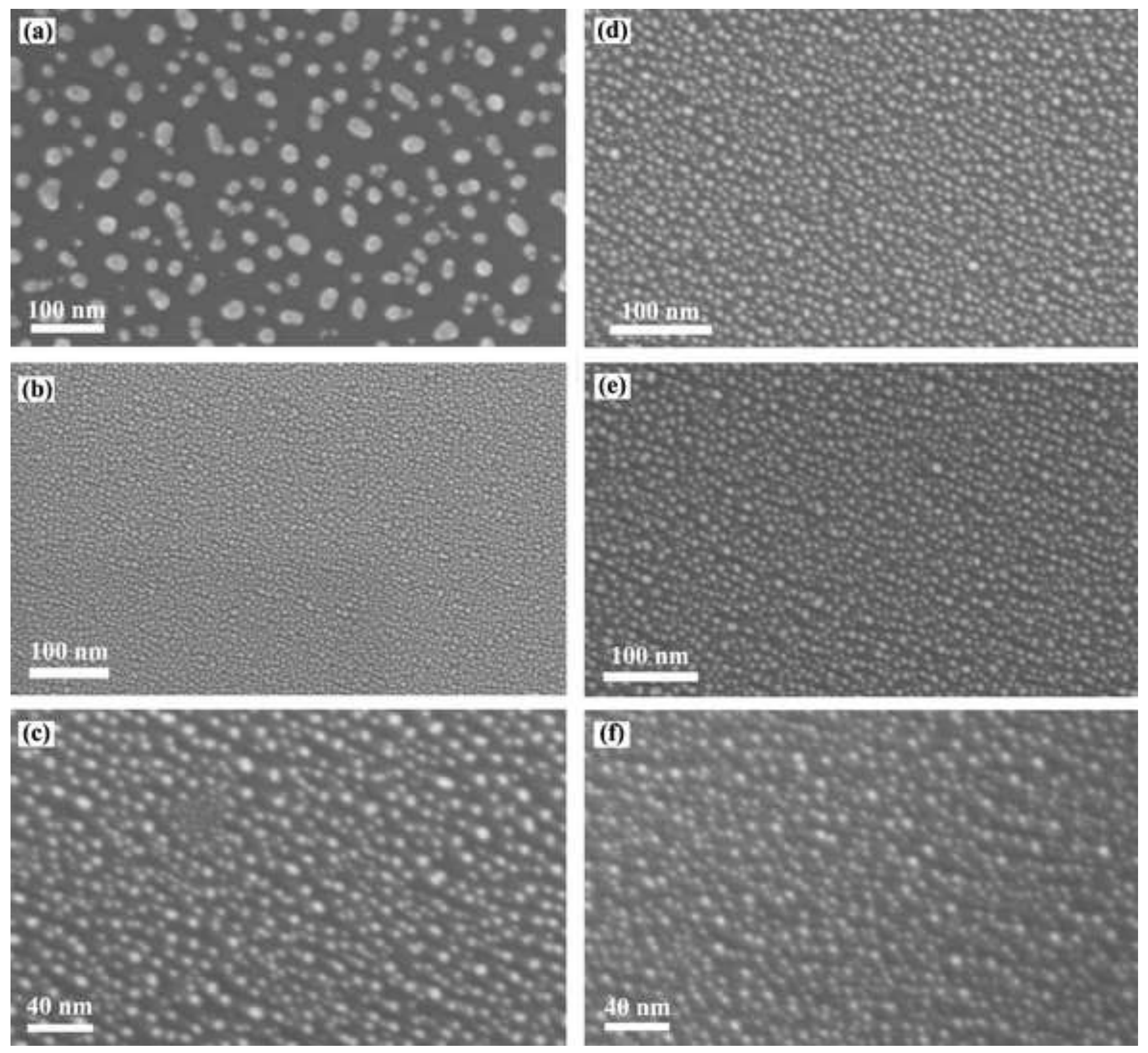

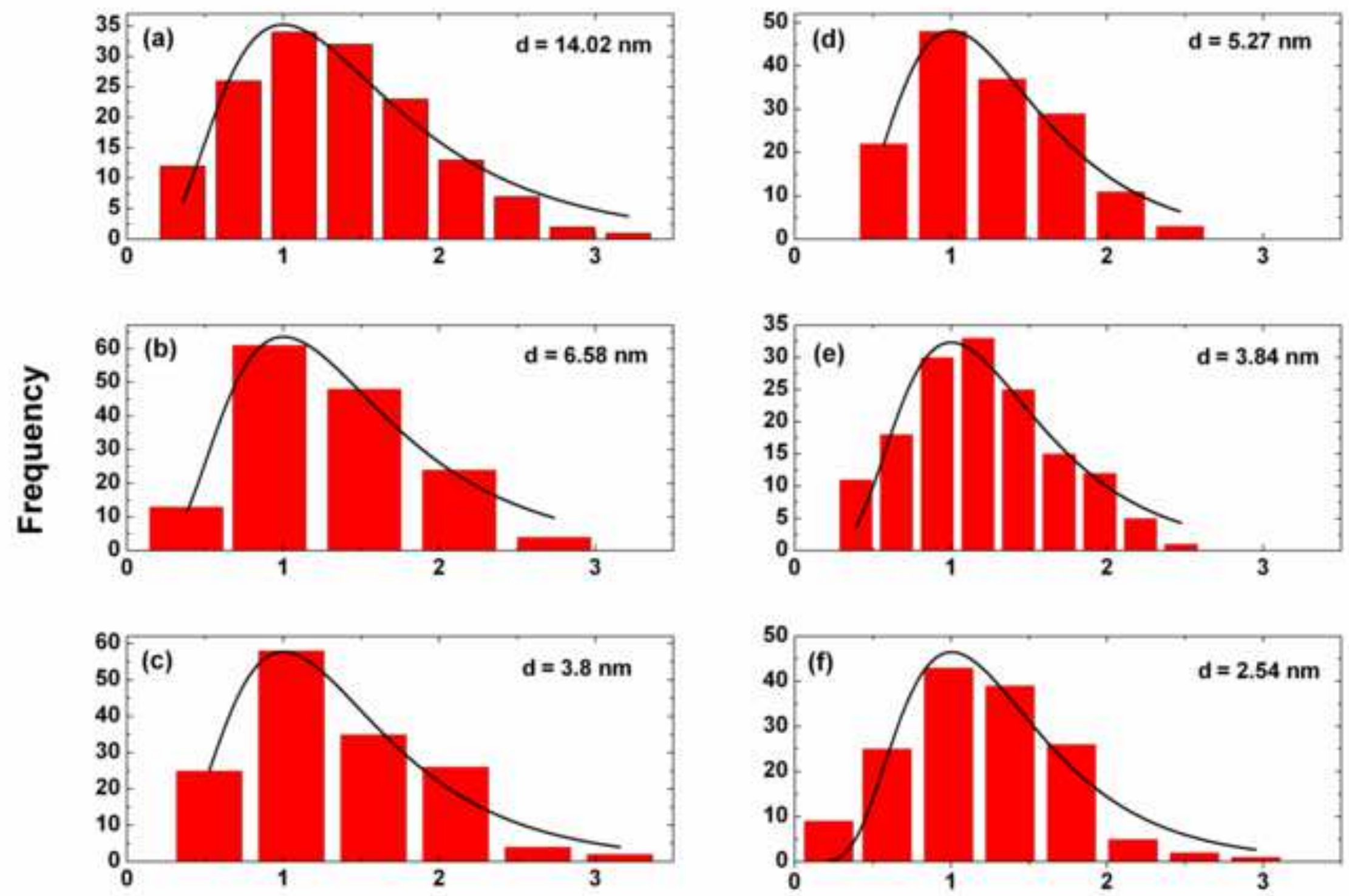

Grain diameter (normalized) 


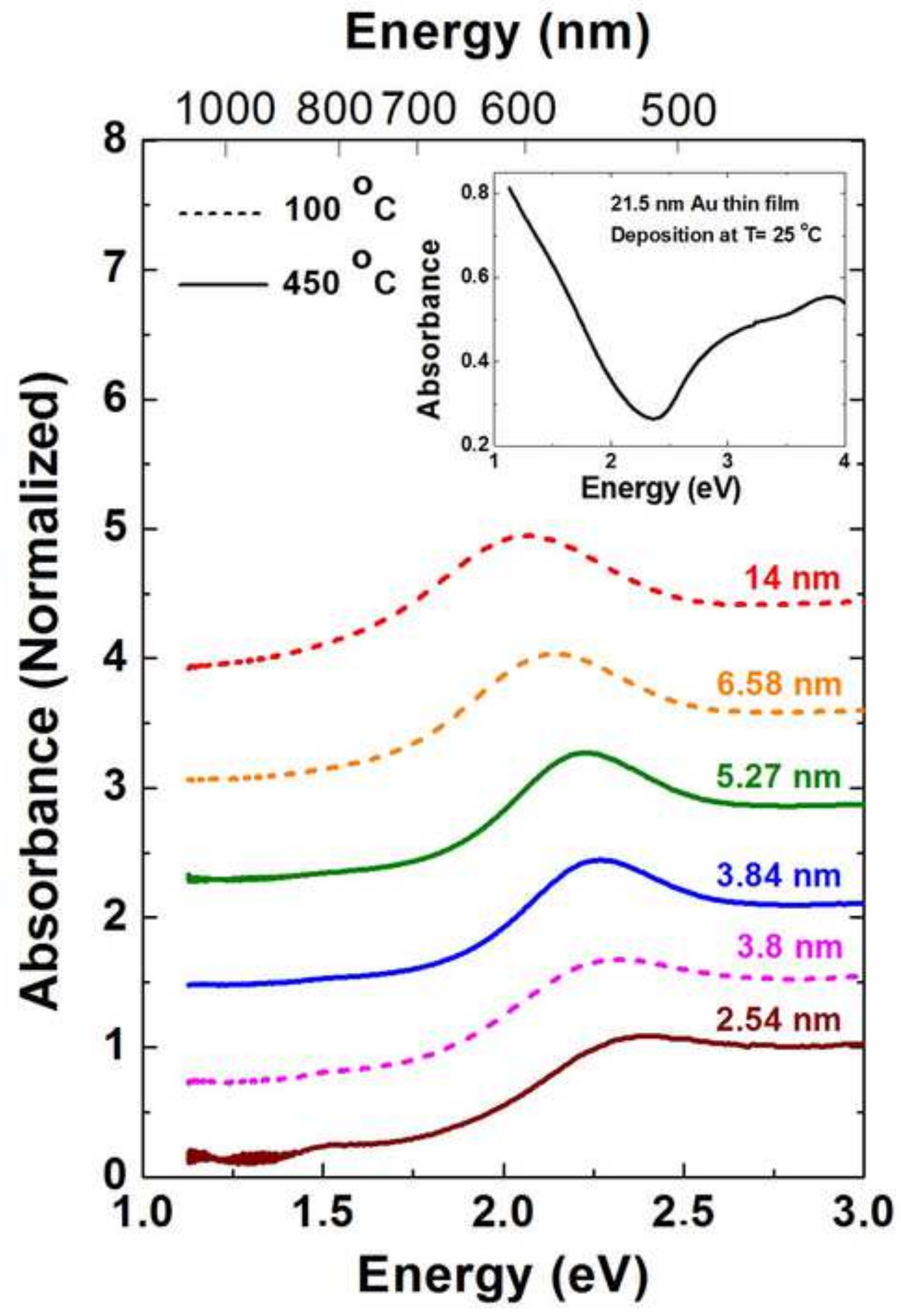




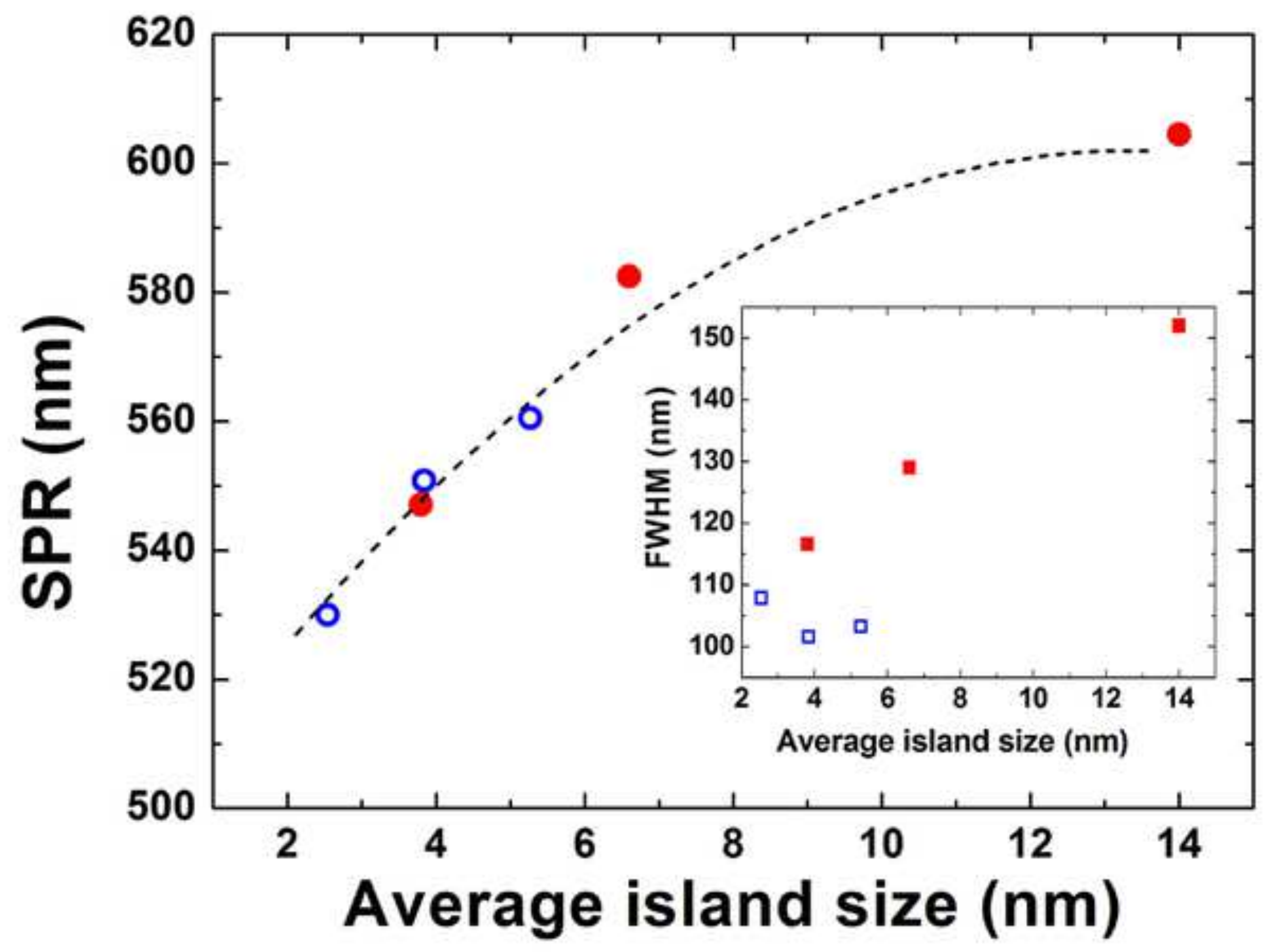




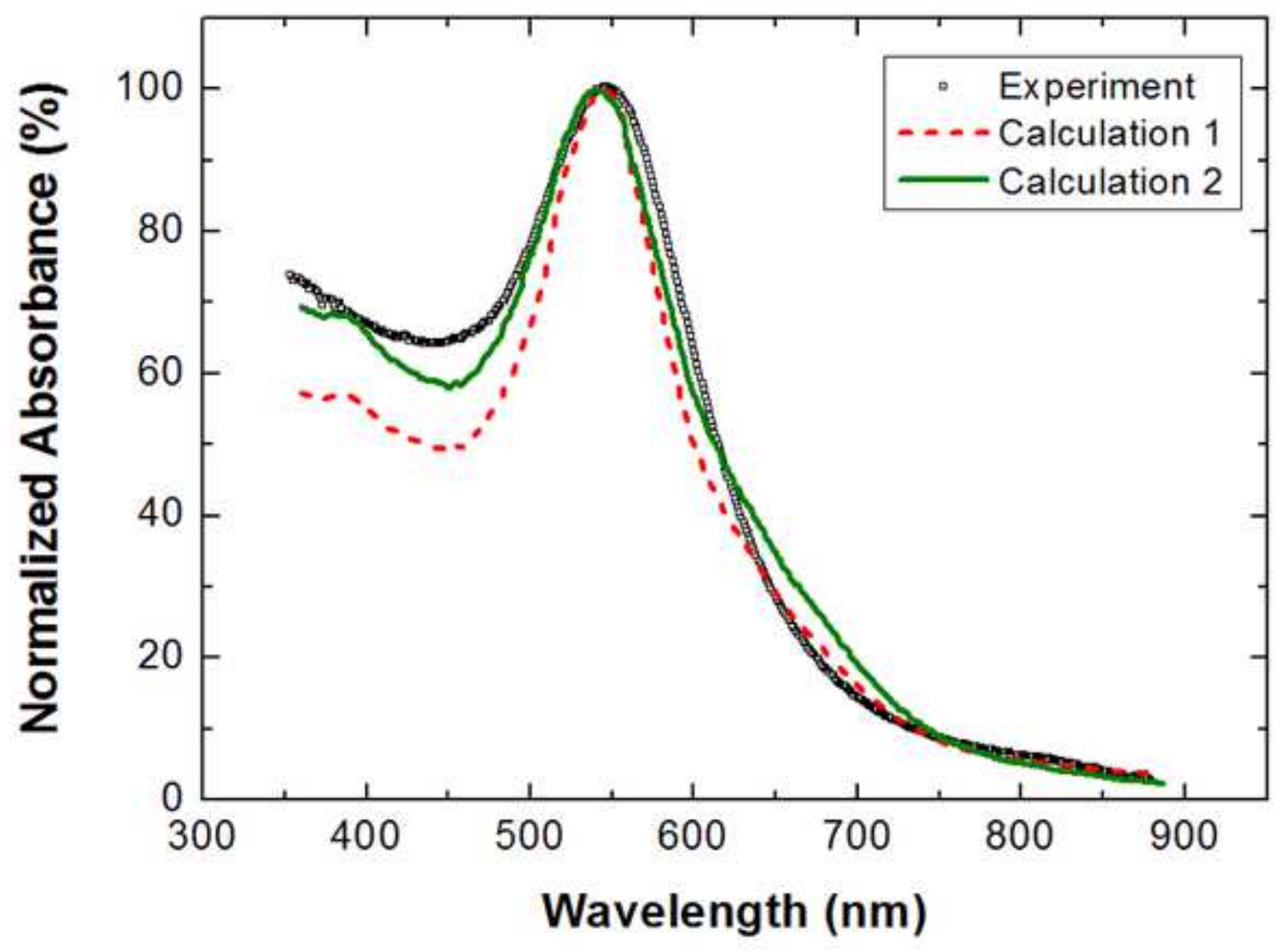



Supplementary material (audio/video files etc)
Click here to download Supplementary material (audio/video files etc): Gramm_JNR_revised_highlighted.doc

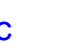

lick here to download Supplementary material

(

(1)

(1)

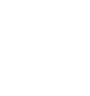

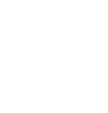

